\title{
La procédure de conclusion des acords inter- nationaux au Brésil
}

\author{
Vicente Marotta Rangel \\ Docente Livre de Direito Internacional Pu- \\ blico na Faculdade de Direito da Universidade \\ de São Paulo.
}

1. Pays très étendu, d'une superficie supérieure à 8.500.000 kms2 (huit millions cinq cent mille kilomètres carrés), le Brésil comprend une population d'environ 65 millions d'habitants. On y trouve des zones de climat soit équatorial soit tropical et même subtropical; les régions géo-économiques se multiplient; la production est hétérogène; les populations diffèrent; la densité démogrâphique oscille beaucoup d'une province à l'autre; les coutumes ne sont pas les mêmes car elles pourraient difficilement se maintenir eu égard à l'extension du territoire. D'autre part, la forme longitudinale du territoire détermine, par elle-même, l'autonomie des grandes divisions.

De l'Indépendance à la proclamation de la République, c'est-á-dire, pendant la période où l'on a adopté un gouvernement monarchique (1822-1889), le Brésil s'est maintenu unitaire. L'abdication de Don Pedro II a coîncidé avec l'institution d'une structure fédérative maintenue d'ailleurs par toutes les Constitutions républicaines (1891, 1934, 1937 et 1946). En raison des facteurs déjà mentionnés, cette 
structure répond mieux aux exigences politiques, économiques et sociales du pays.

Le Brésil est donc un Etat fédéral. L’Union comprend, outre le District Fédéral, 5 Territoires et 21 Etats-Membres. À l'exception du Territoire de Fernando Noronha, les Territoires sont représentés à la Chambre des Députés, un des organes du Parlement National. Les Etats, au contraire, sont représentés tant à la Chambre qu'au Sénat. Ils possèdent les attributs d'unité fédérée. Ils sont autonomes. Chaque Etat a sa propre constitution et élit son Gouverneur et son Parlement.

2. Comme le prescrit la Constitution Fédérale (du 18 septembre 1946), le Brésil maintient, sous le régime représentatif, la Fédération et la République. Tout pouvoir émane du peuple et en son nom sera exercé (art. ler). Le suffrage est universel, direct et obligatoire.

Indépendants et en harmonie, le Pouvoir Exécutif, le Législatif et le Judiciaire sont les Pouvoirs de l'Union. Aucun d'eux n'a le droit de déleguer ses attributions.

Le Président de la République détient le Pouvoir Exécutif. Il doit être âgé de 35 ans au moins. Il est élu par suffrage populaire pour un mandat de 5 ans, et non rééligible pour la période consécutive.

Le Pouvoir Législatif est confié au Sénat et à la Chambre des Députés. Chaque Etat est représenté par trois sénateurs, élus pour huit ans. La Chambre des Députés est formée par 304 membres, élus pour quatre ans proportionnellement au nombre d'habitants des Etats et des Territoires.

Le Pouvoir Judiciaire est exercé par le Suprême Tribunal Fédéral (11 membres nommés à vie par le Président de la République avec l'approbation du Sénat), par le Tribunal Fédéral de Recours ( 9 magistrats nommés de la mểme manière), par des juges et des tribunaux militaires, des juges électoraux et des juges compétents en matière de droit de travail. 
3. Il appartient exclusivement à l'Union de maintenir des relations avec des Etats étrangers et de conclure avec eux des traités et des conventions. Cette disposition consacrée par l'art. 5, n. I, de la Constitution Fédérale est traditionnelle dans le droit public brésilien.

4. Dans le cadre de l'Union, le maintien des rapports avec des Etats étrangers est à la charge du Président de la République, qui, dans le régime présidentiel (en vigueur au Brésil), est à la fois chef d'Etat et chef du Pouvoir Exécutif. Ceite compétence est exclusive. Ainsi l'ont uniformément établi les Constitutions républicaines. Pendant la période monarchique, ces pouvoirs appartenaient à l'Empereur, spécialement celui de nommer des ambassadeurs et de diriger des négociations politiques avec l'étranger.

C'est par l'intermédiaire du Ministère des Affaires Etrangères que le Pouvoir Exécutif maintient des contacts et des échanges avec d'autres Etats; c'est encore par son intermédiaire qu'on négocie des traités internationaux.

5. Cependant, lorsqu'on dépasse la simple phase du maintien des relations internationales ou de la négociation des traités et que l'on fait appel à une affírmation plus profonde de la volonté de l'Etat, alors la compétence du Pouvoir Exécutif n'est plus exclusive. La déclaration de guerre et le rétablissement de la paix ne peuvent se passer de l'autorisation du Congrès National. D'autre part, la conclusion des traités et conventions internationaux recquiert le referendum du Pouvoir Législatif.

Dans notre pays, la formation des trạités, après la phase préliminaire de la négociation, s'apparente à celle des lois. Elle exige outre l'approbation du Congrès, la promulgation et la publication, celle-ci soumise aux normes générales pour la mise en vigueur. 
6. Le principe de la compétence du Parlement dans la procédure de ratification des traités est traditionnel dans les Constitutions républicaines. La Constitution Impériale de 1824 ne le reconnaissait pas. Seuls devraient être soumis au préalable à l'approbation du Pouvoir Législatif (Chambre des Députés et Senat) les traités conclus en temps de paix qui réglaient la cession ou l'échange des territoires de l'Empire, ou des possessions sur lesquelles l'Empire aurait des droits. Les autres traités n'étaient portés à la connaissance des organes parlementaires qu'après avoir été conclus par l'Empereur, et même ainsi, seulement quand l'intérêt et la sûreté de l'Etat le permettaient. La Constitution Républicaine de 1891 a apporté sur ce point un changement radical. Elle disposa, d'une manière semblable d'ailleurs aux Constitutions postérieures, qu'il serait de la compétence majeure du Congrès National de "résoudre en définitive sur les traités et conventions avec les nations étrangères" (art. 34, n. 12).

Dans un commentaire de cette disposition, devenu classique, João Barbalho expliquait (Constituição Federal Brasileira, Comentários, 1902, pg. 110) que, les traités étant un échange de concessions et établissant une reciprocité d'obligations, il n'était pas du ressort du Pouvoir Exécutif d'engager motu proprio la responsabilité de la nation, de lui provoquer des engagements et de lui attirer des charges, même au prix de certains avantages. "Pour cela la ratification des accords, des conventions et des traités passés par le Président de la République, fut reservée au Congrès National. Ce correctif entraîna une garantie contre des abus possibles, contre la mauvaise compréhension et le mépris des plus hauts intérêts nationaux". Et, cherchant à mettre en relief la solution brésilienne en face de Ia nord-américaine, il estimait que, si l'approbation de l'accord international par le seul Sénat était plus pratique et accélerait l'acte d'approbation, il était pourtant indubi- 
table que le procéssus adopté par la Constitution brésilienne (approbation tant par le Sénat que par la Chambre) obéissait plus complètement au principe représentatif démocratique: "et en faisant passer l'affaire par plus d'un creuset, on vise à l'épurer davantage" ("e fazendo o negócio passar por mais de um cadinho, visa apura-lo melhor").

Aux termes de la Constitution Fédérale de 1934, le Pouvoir Législatif avait, de la même façon, le droit exclusif de "décider en définitive sur les traités et les conventions conclus avec les nations étrangères, signés par le Président de la République, y compris ceux concernant la paix" (art. $40 a$ ); d'après cette même Constitution (art. $56, \mathrm{n} .6$ ), et d'une manière cohérente, il appartenait personnellement au Président de la République de "signer des conventions et des traités internationaux ad, referendum du Pouvoir Législatif".

L'actuelle Constitution, du 18 septembre 1946 (art. 66, n. I), établit, de manière analogue, qu'il appartient au Congrès National de décider en définitive sur les traités et conventions passés avec les Etats étrangers par le Présỉdent de la République; et aussi, d'une manière cohérente, elle prescrit, dans l'art. 87, n. VII, que le Président de la République seul a le droit de "passer des traités et des conventions internationaux ad referendum du Congrès National".

Ainsi, conformément à l'actuelle Constitution (et selon d'ailleurs les Constitutions républicaines antérieures), $\mathbf{l} \vec{a}$ conclusion des conventions internationales n'a lieu qu'après avoir reçu, en vue de la ratification, l'autorisation du Parlement, c'est-à-dire, des deux Chambres qui le composent: Sénat et Chambre des Députés. Comme le signale M. Themistocles Brandão Cavalcanti, "les traités et les conventions internationaux sont coñclus par le Pouvoir Exécutif, par l'intermédiaire de ses délegués, ambassadeurs et organes propres, mais leur validité dépend surtout de l'approbation du Congrès qui décide en définitive à leur égard". (A Constituição Federal Comentada, II, pg. 129). 
7. Néanmoins, une question se pose: n'y a-t-il pas d'accords qui se passent de cette approbation, d'accords réputés exclusivement du ressort du Pouvoir Exécutif? La réponse n'est pas unanime. Ce sujet donne encore lieu à des controverses, motivées d'ailleurs par une certaine imprécision du texte de la Constitution, qui, il faut le reconnaître, n'a pas suivi la meilleure technique.

La Constitution se réfère aux "traités et conventions" Qu'a-t-elle voulu dire par ces termes? Signalent-ils deux espèces d'accords pour assurer que tous les accords internationaux ne peuvent se passer de l'approbation parlementaire? Ou alors désignent-ils une certaine catégorie d'accords, des traités ou treaties, pour témoigner l'existence d'une autre catégorie, non mentionnée explicitement dans la Constitution, qui serait celle des accords em forme simplifiée, pour laquelle l'approbation parlementaire ne serait plus recquise?

La Constitution permet la formation de doutes qui ont favorisé des arguments propres aux deux courants d'opínions. C'est que, d'après l'expression d'une partie de la doctrine, l'approbation parlementaire est recquise pour tous les accords internationaux; d'après l'expression de l'autre partie, cette approbation ne l'est pas pour tous les accords.

\section{III}

8. Au premier courant se rattache la tradition des commentateurs des Constitutions républicaines. C'est le cas de Barbalho, déjà cité.

C'est aussi le point de vue de M. Pontes de Miranda. "Le Brésil - écrit-il - ne s'engage que par la ratification; c'est le Congrès National qui rend définitif tout traité, convention ou acte unilateral du droit des gens (art. 66, n. I). Le Président de la République les signe sous cette condition inéluctable" (Comentários à Constituição de 1946, 2. ${ }^{\mathrm{a}}$ ed., vol. II, 1953, pg. 404). 
9. La pensée de Clovis Bevilaqua, internationaliste réputé, ne diffère pas. D'après lui, aucune convention ne peut au Brésil être exécutée "sans l'approbation du Congrès". Tous les accords internationaux sont conclus par le Pouvoir Exécutif ad referendum du Congrès (Direito Público Internacional, 2. ${ }^{a}$ ed., 1939, pg. 21).

10. "According to the terms of the Brasilian Constitution, - écrit plus récemment M. José Sette. Camara - the Congress has the last word as to whether the country will or will not be engaged in an international compact" (The Ratification of International Treaties, The Ontario Publishing Company Limited, Toronto, 1949).

11. C'est aussi ce que Braz de Sousa Arruda, Luis Antonio da Gama e Silva et Nicolau Nazo enseignent actuellement dans leurs cours universitaires.

12. De la même façon que Lafayette Rodrigues Pereira, M. Haroldo Valladão, après un examen minutieux du sujet, reconnait (Aprovação de Atos Internacionais pelo Congresso Nacional, Parecer, Boletim da Sociedade Brasileira de Direito Internacional, n. 11-12, pgs. 95-108) que la règle, d'après le droit brésilien, est celle de la nécessité de l'approbation par le Congrès des accords internationaux, à une seule exception: "celle qui concerne les pactes négociés par des chefes militaires dans les limites de leurs attributions".

13. M. João Hermes Pereira de Araujo signale que la Constitution de 1946, comme celle de 1934, établit qu'il appartient en propre au Président de la République de passer des "conventions et des traités internationaux, ad referendum du Pouvoir Législatif". La Constitution de 1891 disait (art. 48) qu'il était de la compétence du Président de régler "des accords, des conventions et des traités, toujours ad referendum du Congrès". Les Constitutions postérieures omirent les termes "toujours" et "accords". Pour cette raison et aussi par l'exigence d'une règle consuétudinaire, il semble à cet auteur que constitueraient une ex- 
ception à la règle de la nécessité de l'approbation parlementaire "les actes internationaux dont la mátière est du ressort du Pouvoir Exécutif" (A Processualística dos Atos Internacionais, Rio de Janeiro, 1958, pgs. 172-173).

14. M. Levi Carneiro étend ces exceptions aux accords suivants: a) accords dont la matière est de la compétence exčlusive du Pouvoir Exécutif, et qui ne soient pas d'une importance capitale; b) accords sur des sujets d'importance secondaire, en vue de l'exécution, de l'application ou de l'élucidation d'un doute; c) ceux de modus vivendi, visant seulement à maintenir un état de fait ou établissant les bases de négociations futures; d) ceux de prorogation ou de modification d'un accord exécutif conclus par échange de notes, encore en vigueur; e) ceux qui promettent la réciprocité en cas d'extradition (Acordos por Troca de Notas e Aprovação pelo Congresso Nacional, Parecèr, Boletim da Sociedade Brasileira de Direito Internacional, n. 13-14, 1951, pgs. 129-142).

15. D'autre part, M. Hildebrando Accioly fait remar̃quer que les hypothèses d'accords internationaux qui peuvent se passer de l'approbation parlementaire sont diverses, a savoir: a) accords sur des sujets de la compétence exclusive du Pouvoir Exécutif; b) ceux conclus par des intermédiaires ou des fonctionnaires compétents en matière d'intérêt local ou d'importance restreinte; e) ceux qui consignent simplement l'interprétation des clauses d'un traitẻ déjà en vigueur; d) ceux qui découlent, d'une façon logique et nécessaire, de quelque traité déjà en vigueur et en sont comme le complément; e) ceux de modus vivendi, lorsqu'on a seulement en vue de maintenir un état de fait ou d'établir de simples bases en vue de négociations futures.

M. Accioly dit que fréquemment s'ajoutent à ces cas d'autres comme, par exemple, ceux qui concernent la prorogation des traités avant leur expiration, les déclarations dites d'extradition, c'est-à-dire les promesses de réciprocité en matière d'extradition, faites par simple échange de notes (A Ratificação e a Promulgação dos Tratados em face da 
Constituição Federal Brasileira, Boletim, da Sociedade Brasileira de Direito Internacional, n. 7, 1948, pg. 8).

Le même auteur affirme qu'au Brésil "depuis de nombreuses années la coutume - quoiqu'on la prétende établie extra legem - est de ne pas exiger l'approbation du Congrès National pour certains actes internationaux" (Hildebrando Accioly, Ainda o Problema da Ratificação dos Tratados em face da Constituição Federal Brasileira, Boletim, n. 13-14, 1951, pg. 33).

16. L'opinion de M. Geraldo Eulalio de Nascimento e Silva confirme les cinq hypothèses de dispense d'approbation parlementaire, indiquées par Accioly. Comme exemple de la seconde de ces hypothèses, il rappelle les accords que règlent les commandants militaires ou de la marine, en temps de guerre et en cas d'urgence. (Cf. A Referenda feita pelo Congresso Nacional de Tratados Internacionais, in "Direito", vol. XLVI, 1947, pgs. 41-46).

\section{IV}

17 D'après ce que l'on a vu sur la nécessité de l'assentiment parlementaire à tout accord international, la doctrine est incertaine et instable.

La controverse attend toujours l'occasion d'être appréciée et réglée une fois pour toutes par le Pouvoir Judiciaire.

18. Tant que la solution judiciaire n'intervient pas et que la doctrine se révèle de plus en plus imprécise, la pratique tend à favoriser l'adoption d'accords exécutifs. Et elle le fait - d'après ou contre les termes de la Constitution - depuis le régime de la Grande Charte de 1891.

A ce propos, M. Braz de Sousa Arruda témoigne, dans son cours (Faculté de Droit de l'Université de São Paulo, 1960 , pg. 165), il y a de divers accords mis en vigueur dont le Législatif n'a pas pris connaissance. 
19. M. Hildebrando Accioly donne comme exemple les actes bilatéraux suivants signés par le Brésil: ceux du 10 et du 20 juillet 1899 avec l'Allemagne; celui du 9 août 1895 avec l'Argentine; ceux du 4 juin et du 24 septembre 1891 avec l'Autriche-Hongrie; ceux du 4 et du 9 juillet 1899 avec la Belgique; ceux du 10 mai 1895 et du 30 octobre 1899 avec la Bolivie; celui du 10 janvier 1896 avec le Chili. Il en ajoute d'autres plus récents, conclus sous le régime des Constitutions de 1934 et de 1946, respectivamente: accords commerciaux bilatéraux de 1936 conclus avec l'Allemagne, l'Autriche, le Chili, la Colombie, Cuba, Danemark, Equateur, Mexique, Norvège, Pérou, Roumanie, Suisse, Tchécoslovaquie; accords bilatéraux sur l'instruction militaire, signés soit avec les États-Unis soit avec la France en 1936; accords sur le riz, conclus le 23 décembre 1946, avec les États-Unis et avec la Grande-Bretagne; accords bilatéraux sur les tissus de coton passés en 1946 et en 1947 avec l'Uruguay, le Chili, la Bolivie, le Paraguay; accord du 26 novembre 1948, avec les États-Unis en vue de l'étude et de la jouissance des ressources minérales du Brésil; accords sur les valises diplomatiques, conclus en 1951 et en 1952, avec la Colombie, le Costa-Rica et le Honduras; accord du 15 octobre 1951, avec le Portugal, en vue de la suppression de visas aux passeports; arrangements comerciaux avec l'Islande, l'Espagne, l'Italie et la Grèce, passés en 1952; accord administratif sur les payements avec l'Islande, l'Espagne, l'Italie, la Grèce, conclus en 1952; accord administratif sur les payements avec le Japon, conclu le 12 septembre 1952, etc. etc. (Ainda o Problema da Ratificação dos Tratados em Face da Constituição Federal Brasileira, op. cit., pgs. 22-28).

20. À une solution judiciaire serait préférable celle qui proviendrait du législateur.

Les incertitudes nées de l'imprécision du texte constitutionnel sont de sérieuses atteintes à la sécurité, au dynamisme et à la rigueur avec lesquels les organes gouverne- 
mentaux doivent agir dans le cadre complexe et très important des rapports internationaux.

Jusqu'à ce que le législateur intervienne efficacement, il n'y aura d'autre solution - suggère M. Themistocles Brandão Cavalcanti - que de soumettres tous les actes internationaux au Congrès en vue de la ratification. "Il serait sans doute dangereux en vertu des seuls principes doctrinaires d'accorder au Président de la République un pouvoir de discrimination des actes internationaux" (A Constituição Federal Comentada, vol. II, 2. ${ }^{\mathrm{a}}$, ed. 1952, op. cit., pg. 131).

"Et il ne serait pas licite de laisser à l'arbitre du Pouvoir Exécutif sans contestation - écrit plus loin le même juriste - des intérêts d'une telle importance alors que des mesures d'ordre intérieur de moindre importance ne sont pas soumises au Congrès" (pg. 263).

21. Corriger la lacune et le doute de la Constitution pourrait être l'oeuvre soit du législateur ordinaire (hypothèse retenue par M. Brandão Cavalcanti), soit du législateur constituant. Parmi ceux qui soutiennent expressément cette dernière hypothèse, se trouvent des juristes appartenant aux courants divergents déjà mentionnés.

Ainsi, d'une part, écrit M. Pereira de Araujo, la réforme aurait lieu en vue de la reconnaissance de normes coutumières "en faveur du progrès de nos relations internationales" (op. cit., pg. 173).

D'autre part, la réforme devrait éviter que des actes internationaux non classés parmi les conventions et les traités fussent “dispensés du contrôle du Législatif" (Afonso Arinos de Melo Franco, Estudos de Direito Constitucional, Rio, 1957, pgs. 265-266).

\section{$\mathrm{V}$}

22. Une fois approuvé par le Congrès, le traité n'est plus soumis à la sanction du Président de la République (comme il l'était d'après la Constitution de 1891) mais 
promulgué - par décret législatif — par le Président du Senat, car il s'ágit d'une matiére du ressort exclusif du Congrès (arts. 66 et 71 de la Constitution Fédérale de 1946).

L'effet de cette promulgation est de permettre au Président de la République de ratifier le traité et d'échanger ou de déposer les instruments de ratification.

23. C'est après l'échange ou le dépôt des ratifications que le Président de la République - autorité en droit interne des accords conclus internationalement - doit rendre un acte espécial pour qu'ils soient aplicables en Droit interne.

Cet acte est le décret de promulgation du traité.

Bien qu'elle ne soit pas prévue dans un texte légal, la promulgation est en usage depuis 1826, lors du premier traité conclu par l'Empire du Brésil.

24. Le décret de promulgation, de même que le texte intégral du traité qui l'accompagne, sont publiés au Journal Officiel.

Sauf disposition contraire, le traité de même que la loi entre en vigueur dans le territoire brésilien quarante cinq jours après sa publication, délai présumé suffisant pour que le norme juridique soit portée à la connaissance de tous (art. ler. de la Loi d'Introduction au Code Civil Brésilien).

\section{VI}

25. Les accords internationaux n'entrent dans le droit interne qu'avec une valeur supérieure à celle des lois ordinaires.

C'est ce que soutient la doctrine dominante. Ainsi: Pedro Lessa, "Do Poder Judiciário", Rio, 1915, pg. 220.

Philadelpho Azevedo, "Os Tratados e os Interêsses Privados em face do Direito Brasileiro, Boletim da Sociedade Brasileira de Direito Internacional", 1945, ano I, n. 1, pgs. 12-29. 
Vicente Rao, "O Direito e a Vida dos Direitos", $1 .^{\circ}$ vol., tomo II, 1952 , pg. 489.

Hildebrando Accioly, "Tratado de Direito Internacional Público", I. 2. ${ }^{a}$ ed., 1956, pgs. 44-50, pg. 547.

Oscar Tenório, "Direito Internacional Privado", 3. ${ }^{\text {a }}$ ed., 1953 , pg. 55 .

Carlos Maximiliano, "Comentários à Constituição Brasileira" 4. ${ }^{\mathrm{a}}$ ed., 1948, n. 353.

Carlos Medeiros Silva, "As Atribuições Constitucionais do Poder Executivo", in Revista de Direito Administrativo, vol. 31, 1953, pg. 8. chaire.

C'est ce que Braz de Sousa Arruda enseigne dans sa

Telle est aussi l'orientation de la jurisprudence interne. C'est ce que Philadelpho Azevedo a essayé de montrer dans un commentaire de deux arrêts du Suprême Tribunal Fédéral. Cette orientation fut, d'ailleurs, confirmée par la suite par le même Tribunal, d'après la Revue de Droit Administratif, 1953, vol. 34, pgs. 106-110.

À propos de la jurisprudence de ce Tribunal, Philadelpho Azevedo précise que'elle se prononce en faveur du prestige attaché à la parole du pays, consignée dans des Traités, qui sont ainsi exclus de la procédure commune de révocation ("Os Tratados e os Interêsses Privados", op. cit., pg. 28).

26. Les Etats - écrit Clovis Bevilaqua - doivent observer les traités qu'ils ont régulièrement conclus. Il ajoute que la "validité des conventions ne dépend pas des changements constitutionnels subis par les Etats contractants" ("Direito Público Internacional", 2. a ed., 1939, §§ 167 et 168 ).

La majorité des auteurs enseigne, cependant, que les accords internationaux ne peuvent pas prévaloir sur la Constitution Fédérale. C'est ce que soutiennent Pedro Lessa, "Do Poder Judiciario", op.cit., pg. 224; Carlos Medeiros 
Silva, "As Atribuições Constitucionais do Poder Executivo", op. cit., pg. 6; Aurelino Leal, "Teoria e Prática da Constituição Federal Brasileira", parte primeira, pg. 628; Pontes de Miranda, "Comentários à Constituição", I, pg. 253; Eduardo Espinola e Eduardo Espinola Filho, "Tratado de Direito Civil Brasileiro, vol. VII, 1941, pg. 571.

Ce dernier auteur ånne un exemple: les traités signés par le Brésil, accordant l'extradition des nationaux, ont cessé d'être en vigueur d'après les Constitutions de 1934, 1937 et 1946, qui l'ont expréssement défendus.

M. Gama e Silva insiste: "Aucun traité international ne peut s'opposer aux normes constitutionnelles, car le précepte de la primauté des lois constitutionnelles sur leurs, "la loi ne peut exclure de l'appréciation du Pouvoir Internacional Privado, Curso, pg. 141).

\section{VII}

27 À la rigueur, l'interprétation en Droit interne des accords qui y sont applicables a lieu non par voie gouvernementale (administrative) mais par voie juridictionnelle. D'après la Constitution brésilienne (art. 141, $\S 4 .^{\circ}$ ) d'ailleurs, "la loi ne peut exclure de l'appréciation du Pouvoir Judiciaire aucune atteinte au droit individuel" Il appartient à ce Pouvoir de contrôler la constitutionnalité des traités, car on assimile ceux-ci aux lois. Ce contrôle est d'autant plus fort du fait que, d'après la Loi d'Introduction au Code Civil, "le Magistrat ne refusera pas de juger, sous prétexte du silence, de l'obscurité ou de l'insuffisance de la Ioi".

Le juge applique le traité dûment ratifié et publié comme il applique la loi brésilienne. Si les parties trouvent qu'il a fait une mauvaise application ou interprétation des traités, elles pourront faire usage de voie de recours du droit commun.

Il appartient au Suprême Tribunal Fédéral de connaitre et de juger ces recours en dernière instance. Il jugera 
"em recurso ordinario" les causes réglées par des juges locaux, basées sur un traité international. Et il jugera "em recurso extraordinario" les causes décidées dans une seule ou dernière instance par d'autres tribunaux ou juges, quand la décision est contraire à la lettre du traité international (art. 101, n. II, $b$, e n. III, $a$, de la Constitution).

28. Les doutes jadis suscités sur la possibilité de l'examen de la constitutionnalité d'un traité par le Pouvoir Judiciaire, comme s'il s'agissait d'une loi ordinaire, n'existent plus. La solution en faveur de ce contrôle est deveñue bien établie, comme le certifièrent Pontes de Miranda (Comentários, pg. 142) et Aurelino Leal (Op. cit., pg. 629), dont les enseignements furent mentionnés par Philadelpho Azevedo, quand il fut ministre du Suprême Tribunal Fédéral (Archivo Judiciario", vol. 69, 1944, pg. 19 - Apelação Civil n. 7872).

Les conventions ne seraient soustraites au judicial control que dans l'hipothèse où le Pouvoir Judiciaire devrait prendre connaissance des questions exclusivement politiques, comme par exemple celles qui toucheraient l'opportunité, l'exactitude ou la convenance de ces actes. Actes qui sont entre autres, cités par Rui Barbosa: la déclaration de guerre et la conclusion de la paix; le maintien et l'orientation des relations internationales; le contrôle des pouvoirs des représentants des gouvernements étrangers; la reconnaissance de l'indépendance, de la souveraineté, et du gouvernement des autres Etats; la fixation des frontières du pays avec ses voisins; la conclusion et la rescision des conventions; le régime $d u$ commerce international ("O Direito do Estado do Amazonas ao Acre Setentrional”, vol. I. Rio, 1910, pg. 163).

Le contrôle judiciaire subsistera en cas d'abus de pouvoir ou si la question cesse d'être exclusivement politique.

Ainsi, le Judiciaire décidera sur les questions où l'on prétend que le traité a modifié - écrit M. Pontes de Miranda - "ce que la Constitution a établi sur la compétence législative ou financière des Etats-membres, ou sur Ies 
droits individuels" (Comentários à Constituição de 1946, vol. II, 2. ${ }^{a}$ ed., pg. 470). Toutes les fois que la question politique se lie à des actes qui violent des droits, laissant d'être ainsi exclusivement politique. Toutes les fois que l'on discute - ajoute le même juriste - si l'acte du Pouvoir Exécutif, du Pouvoir Judiciaire ou du Pouvoir Législatif, est constitutionnel ou non, la question judiciaire est formulée, l'èlément politique est dépassé et on est tombé sur le terrain de la question juridique (pg. 474).

\section{VIII}

En vertu de ce que nous venons d'exposer, nous pouvons dégager les conclusions suivantes:

1. ${ }^{\circ}$ Il appartient exclusivement à l'Union de maintenir des rapports avec des Etats étrangers et de conclure avec eux des traités et des conventions. Cette disposition -- consacrée par l'art. 5, n. I, de la Constitution - est traditionnelle dans le droit public brésilien.

2. ${ }^{\circ}$ Dans le cadre de l'Union, le maintien des relations avec les Etats étrangers est à la charge du Président de la République, qui, dans le régime présidentiel (en vigueur au Brésil), est à la fois chef d'Etat et chef du Pouvoir Exécutif. Il a la compétence majeure de passer des traités et des conventions internationaux ad referendum du Congrès National (art. 87, n. VII).

3. Il appartient au Congrès National de décider en définitive sur les traités et conventions passés avec l'étranger (art. 66, n. I).

4. D'après l'expression d'une partie de la doctrine, l'approbation parlementaire est requise pour tous les accords internationaux; d'après l'expression de 
l'autre partie, cette approbation ne l'est pas pour tous les accords.

$5 .^{\circ} \quad$ La jurisprudence n'a pas jusquà ce jour tranché la controverse et sans doute il appartient au législateur ordinaire ou constituant de se prononcer en définitive. Dans la pratique, cette controverse est source de lacunes et d'incertitudes qui réclament une solution prompte et énergique.

6. La convention internationale étant approuvée par le Congrès, elle est ensuite ratifiée par le Président de la République. L'instrument de ratification étant échangé ou déposé, la convention est promulguée par décret du Président de la République Elle entre en vigueur dans le territoire brésilien, sauf disposition contraire, quarante cinq jours après sa publication au Journal Officiel.

7. ${ }^{0}$ L'orientation dominante de la doctrine et de la jurisprudence est que les accords internationaux n'entrent dans le droit interne qu'avec une valeur supérieure à celle des lois ordinaires. La plupart des auteurs estiment que le traité international ne peut pas prévaloir sur la Constitution Fédérale.

8..$^{\circ} \quad$ Linterprétation em Droit interne des accords qui y sont applicables a lieu surtout par voie juridictionnelle. Les conventions n'échappent au judicial control que dans l'hypothèse ou le Pouvoir Judiciaire serait appelé à prendre connaissance des questions exclusivement politiques, comme celles qui concernent l'opportunité, l'exactitude ou la convenance de ces actes. Dès que la question politique touche à une question juridique, le Pouvoir Judiciaire en décide.

Ces conclusions s'appuient sur la manifestation de la doctrine et de la jurisprudence concernant les principales 
dispositions légales qui règlent la procédure de conclusion des accords internationaux au Brésil. Il est clair qu'on doit les considérer, d'autre part, comme de simples prémisses de conclusions à tirer, tout en ayant en vue des solutions d'une plus haute portée pour le pays.

C'est ce que l'on prétend établir à une autre occasion.

\section{Bibliographie}

Accioly (Hildebrando) - A ratificação e a promulgação dos tratados em face da Constituição Federal Brasileira, Boletim da Sociedade Brasileira de Direito Internacional, 1948, n. 7. - Aprovação de Ajustes Internacionais pelo Congresso Nacional, Parecer, Boletim, 1950, ns. 11-12. - Ainda o Problema da ratificação dos tratados, em face da Constituição Federal Brasileira, Boletin 1951, ns. 13-14. - Tratado de Direito Internacional Público, 2. ${ }^{\mathrm{a}}$ ed., 1956.

Arruda (Braz) - Direito Internacional Público (Cours polycopié), 1960.

Araujo (João Hermes Pereira de) - A Processualística dos Atos Internacionais, Rio, 1958.

Azevedo (Philadelpho) - Os Tratados e os Interêsses Privados em face do Direito Brasileiro, Boletim, 1945, n. I.

Barbalho (João) - Constituição Federal Brasileira, Rio, 1902.

Barbosa (Ruy) - o Direito do Estado do Amazonas ao Acre Setentrional, Rio, 1910.

Bevilaqua (Clovis) - Direito Público Internacional, 2. ${ }^{a}$ ed., 1939.

Câmara (José Sette) - The Ratification of International Treaties, Toronto, 1949.

Carneiro (Levi) - Acordos por Troca de Notas e Aprovação pelo Congresso Nacional, Boletim, 1951, ns. 13-14.

Cavalcanti (Themístocles Brandão) - A Constituição Federal Comentada, 1952, 2. ${ }^{\mathrm{a}}$ ed., 4 vols.

Espinola (Eduardo) e Espinola Filho (Eduardo) - Tratado de Direito Civil Brasileiro, vol. VII, Rio, 1941.

Franco (Afonso Arinos de Melo) - Estudos de Direito Constitucional, Rio, 1957.

Leal (Aurelino) - Teoria e Prática da Constituição Federal Brasileira.

Lessa (Pedro) - Do Poder Judiciario, Rio, 1915.

Maximiliano (Carlos) - Comentários à Constituição Brasileira, 4. a ed., 1948, 3 vols. 
Miranda (Pontes de) - Comentários à Constituição de 1946, 2. ${ }^{\mathrm{a}}$ ed., 1953, 5 vols.

Nazo (Nicolau) - Direito Internacional Privado (Cours polycopié), 1960.

Silva (Carios Medeiros) - As Atribuições Constitucionais do Poder Executivo, in Revista de Direito Administrativo, vol. 31, 1953.

Silva (Geraldo Eulalio do Nascimento e) - A Referenda pelo Congresso Nacional de Tratados Internacionais, Direito, julho-agosto 1947 , vol. XLVI.

Silva (Luís Antônio da Gama e) - Direito Internacional Privado (cours polycopié), 1957.

Rao (Vicente) - O Direito e a Vida dos Direitos, 1. ${ }^{\circ}$ vol., 1952.

Tenório (Oscar) - Direito Internacional Privado, 3. ${ }^{\mathrm{a}}$ ed., 1953.

Valladão (Haroldo) - Aprovação de Ajustes Internacionais pelo Congresso Nacional, Parecer, Boletim, 1950, ns. 11-12. 